\title{
Prediction of Falls Risk with Six Different Biomarkers in Mild Cognitive Impairment Using
}

\section{Principal Component Analysis}

Anton De Spiegeleer ${ }^{1,2}$, Tahir Masud ${ }^{3}$, Paul Greenhaff ${ }^{1}$, Nele Van Den Noortgate ${ }^{2}$, Mirko Petrovic $^{2}$, Victoria Hood $^{4}$, John

Gladman $^{5}$, Veronika van der Wardt ${ }^{5}$, Rowan Harwood ${ }^{3}$, Victoria Booth $h^{3,5}$

\author{
${ }^{1}$ MRC/Arthritis Research UK Centre for Musculoskeletal Ageing Research, School of Biomedical Sciences, University of Nottingham, \\ Nottingham, UK \\ ${ }^{2}$ Department of Internal Medicine, Faculty of Medicine and Health Sciences, Ghent University, Ghent, Belgium \\ ${ }^{3}$ Department of Health care for Older People, Nottingham University Hospitals NHS Trust, Nottingham, UK \\ ${ }^{4}$ School of Health Sciences, University of Nottingham, Nottingham, UK \\ ${ }^{5}$ Division of Rehabilitation and Ageing, University of Nottingham, Nottingham, UK
}

Introduction

Falls in older people cause much morbidity and societal cost. Early dementia (ED) is a neglected population in falls research, despite mild cognitive impairment (MCI) being a recognised risk factor. As part of ongoing research into falls in ED we compared different physical/cognitive biomarkers as falls predictors, and investigated if they gave independent information.

Methods

We recruited cross-sectionally, 50 older community-dwellers with ED or MCI [MMSE $=19-26$ or MoCA=1325] and classified them, using Principal Component Analysis (PCA), into clusters, based upon similarity on 6 physiological/cognitive tests: Montreal Cognitive Assessment (MoCA), gait speed (GS), Timed Up and Go (TUG), Berg Balance score (BBS), Falls Efficacy Scale International (FES-I) and Physiological Profile Assessment (PPA).

Results

Complete data was available for 44 participants (mean age $=81$, range $=67-93$ years; 21 women). GS, TUG, BBS and FES-I gave similar information and were therefore reduced to one Principal Component; while MoCA and PPA were reduced to another Principal Component (table 1). Figure 1 shows the participants plotted for both Principal Components. Participants were classified into 2 clusters of similar characteristics, which correlated with a history of falls (last 6 months).

Conclusion

These data suggest that to characterize falls risk in older people with ED/MCI, it may be sufficient to choose two tests: one from Principal Component-1 (GS, TUG, BBS, FES-I) and another from Principal Component-2 (MOCA, PPA). Thus a minimum set of biomarkers could predict falls, allowing targeting of falls prevention to those at the highest risk. Larger longitudinal studies are now required to confirm these findings. 


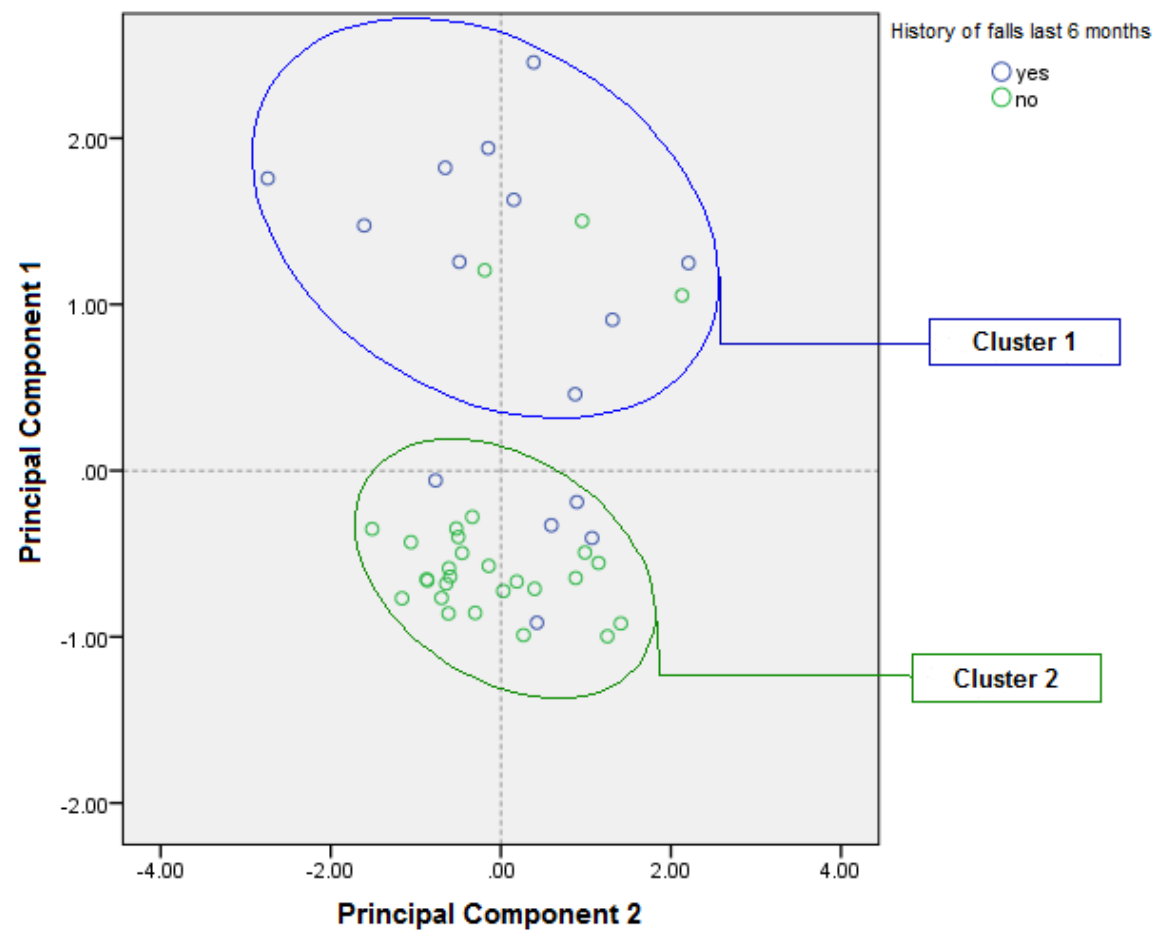

Figure 1. Score plot with PC1 and PC2.

Table 1. Component plot with loading values. ${ }^{*} \mathrm{VAF}=$ Variance Accounted For. Total $\mathrm{VAF}=85 \%$.

\begin{tabular}{|c|c|c|c|}
\hline & \multicolumn{2}{|c|}{ Component } & \multirow[b]{2}{*}{ VAF* } \\
\hline & 1 & 2 & \\
\hline FES & .826 & .185 & 0.72 \\
\hline MOCA & -.037 & -.921 & 0.85 \\
\hline Gait speed & -.926 & -.185 & 0.89 \\
\hline BERG & -.952 & -.071 & 0.91 \\
\hline TUAG & .931 & .222 & 0.92 \\
\hline PPA & .323 & .835 & 0.80 \\
\hline Eigenvalue & 3.4 & 1.7 & 0.85 \\
\hline
\end{tabular}

